Please do not remove this page

RMIT

UNIVERSITY

\title{
Labeled multi-Bernoulli tracking for industrial mobile platform safety
}

Rathnayake, Tharindu; Hoseinnezhad, Reza; Tennakoon, Ruwan; Bab-Hadiashar, Alireza

https://researchrepository.rmit.edu.au/esploro/outputs/9921858864801341/filesAndLinks?institution=61RMIT_INST\&index=null

Rathnayake, T., Hoseinnezhad, R., Tennakoon, R., \& Bab-Hadiashar, A. (2017). Labeled multi-Bernoulli tracking for industrial mobile platform safety. 2017 IEEE International Conference on Mechatronics, 1-6. https://researchrepository.rmit.edu.au/discovery/fulldisplay/alma9921858864801341/61RMIT_INST:Resea rchRepository

Document Version: Accepted Manuscript

Repository homepage: https://researchrepository.rmit.edu.au

(C) 2017 IEEE

Downloaded On 2023/04/26 23:59:09 +1000

Please do not remove this page 


\title{
Labeled Multi-Bernoulli Tracking for Industrial Mobile Platform Safety
}

\author{
Tharindu Rathnayake Reza Hoseinnezhad Ruwan Tennakoon Alireza Bab-Hadiashar \\ RMIT University, Victoria 3083, Australia \\ Emails: s3471339@student.rmit.edu.au, \{rezah,ruwan.tennakoon,abh\}@rmit.edu.au
}

\begin{abstract}
This paper presents a track-before-detect labeled multi-Bernoulli filter tailored for industrial mobile platform safety applications. We derive two application specific separable likelihood functions that capture the geometric shape and colour information of the human targets who are wearing a high visibility vest. These likelihoods are then used in a labeled multi-Bernoulli filter with a novel two step Bayesian update. Preliminary simulation results evaluated using several video sequences show that the proposed solution can successfully track human workers wearing a luminous yellow colour vest in an industrial environment.
\end{abstract}

\section{INTRODUCTION}

Industrial mobile platforms are of universal application within various manufacturing plants. These come in various types such as forklifts, electric buggies, boom and scissor lifts and construction cranes. These multi-tonne machines are capable of inflicting severe injuries, even death. One of the widely used such machines is forklift and we specifically focus on forklift safety in this study. According to our analysis (published in [31]), there were approximately 2500 reports of forklift accidents alone in Victoria during 1997-2013. It also showed that during the past decade, the frequency of the collision incidences are rather consistent and there has been no statistically significant decline. This shows that there is a clear need for intelligent systems that could prevent mobile industrial platforms from striking/crushing pedestrians and other objects.

One of the first attempts to improve the safety was to introduce predefined forklift and pedestrian paths. However, these procedural changes often come at the expense of maintaining a productive work environment [30]. Smarter solutions for industrial mobile platform safety that exploits the benefits of sensing and machine intelligence has been the focus of many works reported in safety, applied signal processing and multisensor fusion literature.

A strand of solutions developed to enhance mobile platform operational safety was based on creating driver-assist technologies using sensors such as laser scanners and imaging devices [12], such as autonomous forklift driving system based on expensive SICK laser scanners [16]. Radio Frequency Identification systems (RFID) are also proposed to prevent the forklift-pedestrian accidents [15], which often activate unnecessary alarms for pedestrians who in no apparent danger but are within the detectable range of the tag. Utilisation of a cheap camera on board the forklift and achieving rich information about the surrounding environment of the vehicle seems to be among the most efficient solutions for the above mentioned safety problem. Such information can be the result of simultaneous detection and tracking (Track-Before-Detect - TBD) of the moving objects around the vehicle.

Recently, random finite set filters were introduced by Mahler [19] and extended to various types such as Probability Hypothesis Density (PHD) filter [18], Cardinalized PHD (CPHD) filter [37], multi-Bernoulli filter [19], [38], labelled multi-Bernoulli (LMB) filter [29] and the generalised version of LMB filter called Vo-Vo filter [35], [36]. ${ }^{1}$ These filters have been reported to be applied for visual tracking in many applications including our recent works [10], [11], [28].

In very few visual tracking applications, rich prior knowledge is available about both the colour contents and the geometric shape of the targets. In the particular safety application concentrated in this research work, enhanced forklift operational safety via visual tracking, we know in advance that the targets (workers) are wearing a safety vest of particular colour, and that their geometric shape in the image is close to a combination of two ellipses.

This paper presents a new multi-target visual tracking algorithm that effectively uses both colour and geometric information embedded in camera images with an LMB filter. The algorithm propagates an LMB multi-target distribution in which each single target state is comprised of the parameters of two ellipses, thus exploiting shape information. The application-specific LMB filter developed in this work includes a two-step update, one for embedding colour information (using colour histograms) and the other for geometric information (using edge detection). Detailed formulation of separable likelihood functions for each step is also presented. The overall TBD filtering scheme is computationally cheap and results in high tracking accuracy. Preliminary simulation results demonstrate that the proposed method can successfully track multiple people moving in an industrial environment. To the best of our knowledge, this is the first time a tracking algorithm is proposed to be used in mobile industrial platform safety.

\section{BACKGROUND}

This section briefly reviews the fundamental concepts, notations and formulae from the literature. A random finite

\footnotetext{
${ }^{1}$ Vo-Vo filter is also called the Generalised Multi-Bernoulli (GLMB) filter. We follow Mahler who called it Vo-Vo filter in his recent book [20].
} 
set is simply a set with a random number of elements and the elements themselves are also random. These elements corresponds to a spatial point pattern on the space of interest.

\section{A. Notation}

In this paper, single-object states are represented using lower case letters (e.g. $\boldsymbol{x}$ and $x$ ), multi-object states are represented using upper case letters (e.g. $\boldsymbol{X}$ and $X$ ) and the spaces are represented using blackboard bold letters (e.g. $\mathbb{N}, \mathbb{X}$ and $\mathbb{L}$ ). In order to distinguish between labeled and unlabeled entities, we use bold letters to represent labeled entities (e.g. $\boldsymbol{x}$ and $\boldsymbol{X}$ ).

\section{B. Labeled RFS}

We use a unique label to distinguish between the targets. This is accomplished by tagging each state $x \in \mathbb{X}$ with a unique positive integer label $\left(\ell_{t}\right) \in \mathbb{L}=\left\{\alpha_{i}: i \in \mathbb{N}\right\}$. Here $\mathbb{N}$ is the set of positive integers and all the $\alpha_{i}$ 's are distinct [36]. Following the general practice, $\ell_{t}$ is set to be the time stamp at which the target is born.

A labeled RFS with state space $\mathbb{X}$ and discrete label space $\mathbb{L}$ is an $\operatorname{RFS} \boldsymbol{X}$ on $\mathbb{X} \times \mathbb{L}$ such that $\mathcal{L}: \mathbb{X} \times \mathbb{L} \rightarrow \mathbb{L}$ is the projection $\mathcal{L}((x, \ell))=\ell$. The finite subset $\mathbf{X}$ of $\mathbb{X} \times \mathbb{L}$ has distinct labels if and only if $\mathbf{X}$ and its labels $\mathcal{L}(\mathbf{X})=$ $\{\mathcal{L}(\mathbf{x}): \mathbf{x} \in \mathbf{X}\}$ have the same cardinality, which can be mathematically denoted as $\delta_{|\mathbf{X}|} \mathcal{L}(\mathbf{x})=\triangle \mathbf{X}=1$ or $|\mathcal{L}(\boldsymbol{X})|=$ $|\boldsymbol{X}|$.

With the set integral defined in [36] and the unit integration over the labeled multi-object state space, i.e. $\int_{\mathbb{X} \times \mathbb{L}} \boldsymbol{\pi}(\boldsymbol{X}) \delta \boldsymbol{X}=1$, the density of a labeled RFS $\mathbb{X}$ is a function given by

$$
\boldsymbol{\pi}: \mathcal{F}(\mathbb{X} \times \mathbb{L}) \rightarrow \mathbb{R}^{+} \cup\{0\}
$$

1) Labeled multi-Bernoulli (LMB) RFS : A labeled multiBernoulli RFS $\mathbf{X}$ with state space $\mathbb{X}$, label space $\mathbb{L}$ and finite parameter set $\left\{\left(r^{(\varsigma)}, p^{(\varsigma)}\right)\right\}: \varsigma \in \Psi$, is a multi-Bernoulli RFS on $\mathbb{X}$, augmented with labels corresponding to the successful non-empty Bernoulli components, where $\Psi$ is the index set with its components $\varsigma$ are assumed to be statistically independent. The label of a target which correspond to a non empty Bernoulli component $\left(r^{(\varsigma)}, p^{(\varsigma)}\right)$, is given by $\alpha(\varsigma)$, where $\alpha: \Psi \rightarrow \mathbb{L}$ is a 1-1 mapping [35]. The set of unlabeled states is a multi-Bernoulli RFS on $\mathbb{X}$. However, the set of labeled RFS is not a multi-Bernoulli RFS on $\mathbb{X} \times \mathbb{L}$. The LMB density with the above mentioned parameters is given by [35]:

$$
\boldsymbol{\pi}(\boldsymbol{X})=\Delta(\mathbf{X}) 1_{\alpha(\Psi)}(\mathcal{L}(\boldsymbol{X}))[\Phi(\boldsymbol{X} ; \cdot)]^{\Psi}
$$

where

$$
\Phi(\boldsymbol{X} ; \varsigma)= \begin{cases}1-r^{(\varsigma)} & \text { if } \alpha(\varsigma) \notin \mathcal{L}(\boldsymbol{X}), \\ r^{(\varsigma)} p^{(\varsigma)}(x) & \text { otherwise }\end{cases}
$$

in which $x$ is extracted from $\boldsymbol{X}$ by finding the member that its label matches $\alpha(\varsigma)$ and $1_{\alpha(\Psi)}$ is the inclusion function.
Following [29], under the assumption that $\alpha$ mapping is an identity mapping, a compact representation of the above multi-target density can be written as:

$$
\boldsymbol{\pi}(\boldsymbol{X})=\triangle(\boldsymbol{X}) w(\mathcal{L}(\mathcal{X})) p^{\boldsymbol{X}}
$$

where

$$
\begin{aligned}
w(L) & =\prod_{i \in \mathbb{L}}\left(1-r^{(i)}\right) \prod_{\ell \in L} \frac{1_{\mathbb{L}}(\ell) r^{(\ell)}}{1-r^{(\ell)}} \\
p(x, \ell) & =p^{(\ell)}(x) .
\end{aligned}
$$

For the sake of simplicity in notation, we denote the above density by $\boldsymbol{\pi}=\left\{r^{(\ell)}, p^{(\ell)}\right\}_{\ell \in \mathbb{L}}$.

\section{TBD-LMB Propagation}

Let $\mathbb{L}_{k}=\{k\} \times \mathbb{N}$ denote the label space for the targets born at time $k$, and $\boldsymbol{x} \in \mathbb{X} \times \mathbb{L}_{k}$ is the state of a target born at time $k$. The label space for all the targets at time $k$, including all the previous label spaces is denoted by $\mathbb{L}_{0: k}$ and is recursively constructed by $\mathbb{L}_{0: k}=\mathbb{L}_{0: k-1} \cup \mathbb{L}_{k}$.

We denote the image observation acquired at time $k$ by $y_{k}$ and $y_{1: k}$ is used to denote the bulk of image observations acquired up to time $k$. According to [35], the labeled multitarget density can be recursively predicted and updated using Bayes recursion:

$$
\begin{aligned}
& \boldsymbol{\pi}_{k+1 \mid k}\left(\boldsymbol{X} \mid y_{1: k}\right)=\int \boldsymbol{f}_{k+1 \mid k}\left(\boldsymbol{X} \mid \boldsymbol{X}_{k}\right) \boldsymbol{\pi}_{k}\left(\boldsymbol{X}_{k} \mid y_{1: k}\right) \delta \boldsymbol{X}_{k} \\
& \boldsymbol{\pi}_{k+1}\left(\boldsymbol{X} \mid y_{1:(k+1)}\right)=\frac{g_{k+1}\left(y_{k+1} \mid \boldsymbol{X}\right) \boldsymbol{\pi}_{k+1 \mid k}(\boldsymbol{X})}{\int g_{k+1}\left(y_{k+1} \mid \boldsymbol{X}_{k}\right) \boldsymbol{\pi}_{k+1 \mid k}\left(\boldsymbol{X}_{k}\right) \delta \boldsymbol{X}_{k}}
\end{aligned}
$$

where $\boldsymbol{f}_{k+1 \mid k}(\cdot \mid \cdot)$ is the multi-object transition density from time $k$ to $k+1, g_{k+1}\left(\cdot \mid y_{k+1}\right)$ is the multi-object likelihood function at time $k+1$ for the given image observation $y_{k+1}$, and the integrals are set integrals as defined in [36]. Henceforward, for the sake of brevity in notations, we will drop the "given observation parts" $\left(\mid y_{1: k}\right.$ and $\left.\mid y_{1:(k+1)}\right)$ of the density arguments, as the dependence of evolved densities on the past and current observations is obvious.

1) LMB Prediction: In the prediction step, at each time step $k-1$, each labeled target $\boldsymbol{x}_{k-1} \in \boldsymbol{X}_{k-1}$ is propagated in to the next time step according to a target transition model, described in the following. With a survival probability of $p_{S, k}\left(\boldsymbol{x}_{k-1}\right)$, it may take on a new state given by $\boldsymbol{x}_{k}$ with a probability density $f_{k \mid k-1}\left(\boldsymbol{x}_{k} \mid \boldsymbol{x}_{k-1}\right)$ (i.e. Markov shift). Further, it may disappear with a probability $1-p_{s, k}\left(\boldsymbol{x}_{k-1}\right)$. We use labeled multi-Bernoulli RFS to implement the above target transition model as follows. A single target state is modelled using a labeled Bernoulli RFS $\boldsymbol{S}_{k \mid k-1}\left(x_{k-1}\right)$. Its survival probability is given by $r=p_{\boldsymbol{S}, k}\left(\boldsymbol{x}_{k-1}\right)$, while its probability distribution is given by $p(\cdot)=f_{k \mid k-1}\left(\cdot \mid x_{k-1}\right)$. Under the assumption that the targets are non interacting, the labeled multi-target state $\boldsymbol{X}_{k}$ at time $k$ can be written as [18], [19]:

$$
\boldsymbol{X}_{k}=\left[\cup_{\boldsymbol{x}_{k-1} \in \boldsymbol{X}_{k-1}} \boldsymbol{S}_{k \mid k-1}\left(\boldsymbol{x}_{k-1}\right)\right] \cup \boldsymbol{\Gamma}_{k},
$$

where $\Gamma_{k}$ is the labeled multi-Bernoulli RFS of spontaneous births. 
Consider an LMB multi-object density defined in state space $\mathbb{X}$ and label space $\mathbb{L}$, and parametrised by $\boldsymbol{\pi}=$ $\left\{r^{(\ell)}, p^{(\ell)}\right\}_{\ell \in \mathbb{L}}$. Reuter et al. [29, prop. 2] have shown that if the multi-object birth model is an LMB with the same state space $\mathbb{X}$, a label space $\mathbb{B}$ that is disjoint from $\mathbb{L}$ (i.e. $\mathbb{X} \cap \mathbb{B}=\emptyset$ ) and parametrised density $\boldsymbol{\pi}_{B}=\left\{r_{B}^{(\ell)}, p_{B}^{(\ell)}\right\}_{\ell \in \mathbb{B}}$, then the predicted multi-object density is also an LMB with state space $\mathbb{X}$ and label space $\mathbb{L}_{+}=\mathbb{L} \cup \mathbb{B}$. Furthermore, the parameters of the predicted LMB density are $\boldsymbol{\pi}_{+}=$ $\left\{r_{+, S}^{(\ell)}, p_{+, S}^{(\ell)}\right\}_{\ell \in \mathbb{L}} \bigcup\left\{r_{B}^{(\ell)}, p_{B}^{(\ell)}\right\}_{\ell \in \mathbb{B}}$, where

$$
\begin{aligned}
r_{+, S}^{(\ell)} & =\eta_{S}^{(\ell)} r^{(\ell)} \\
p_{+, S}^{(\ell)}(x) & =\left\langle p_{S}(\cdot, \ell) f(x \mid \cdot, \ell), p^{(\ell)}(\cdot)\right\rangle / \eta_{S}^{(\ell)} .
\end{aligned}
$$

Here $p_{S}(x, \ell)$ denote the state-dependent probability of survival of an existing Bernoulli component with label $\ell$, $f\left(x_{k+1} \mid x_{k}, \ell\right)$ denote the single-object transition density, and $\eta_{S}(\ell)=\left\langle p_{S}(\cdot, \ell), p^{(\ell)}(\cdot)\right\rangle$.

2) TBD-LMB Update: In track-before-detect approaches, having an image likelihood function (for a given image observation $y$ and multiple objects $\boldsymbol{X}$ ), with the following separable form is very important.

$$
g(y \mid \boldsymbol{X})=f(y) \prod_{(x, \ell) \in \boldsymbol{X}} g_{y}(x, \ell) .
$$

It was shown in [28, prop. 1] that LMB density is a conjugate prior with a separable likelihood function:

$$
\boldsymbol{\pi}(\boldsymbol{X} \mid y) \propto \Delta(\boldsymbol{X}) w_{y}(\mathcal{L}(\boldsymbol{X}))[p(\cdot \mid y)]^{\boldsymbol{X}}
$$

where

$$
\begin{aligned}
w_{y}(L) & =\left[\eta_{y}\right]^{L} w(L) \\
p(x, \ell \mid y) & =\frac{p^{(\ell)}(x) g_{y}(x, \ell)}{\eta_{y}(\ell)} \\
\eta_{y}(\ell) & =\left\langle p^{(\ell)}(\cdot), g_{y}(\cdot, \ell)\right\rangle .
\end{aligned}
$$

The posterior LMB can be parametrised as $\pi_{\text {updated }}=$ $\left\{r_{\text {updated }}^{(\ell)}, p_{\text {updated }}^{(\ell)}\right\}_{\ell \in L}$ where

$$
\begin{aligned}
r_{\text {updated }}^{(\ell)} & =\frac{r^{(\ell)}\left\langle p^{(\ell)}(\cdot), g_{y}(\cdot, \ell)\right\rangle}{1-r^{(\ell)}+r^{(\ell)}\left\langle p^{(\ell)}(\cdot), g_{y}(\cdot, \ell)\right\rangle} \\
p_{\text {updated }}^{(\ell)}(x) & =\frac{p^{(\ell)}(x) g_{y}(x, \ell)}{\left\langle p^{(\ell)}(\cdot), g_{y}(\cdot, \ell)\right\rangle} .
\end{aligned}
$$

\section{State And Measurement Models}

The main points of novelty in our method are in the way we model single target states (to exploit shape information) and measurement likelihood functions (to utilise all the colour- and shape-related information in the image, with the assumed state model).

\section{A. Target state model}

In applications involving tracking of human targets (who are highly likely to be walking in a stand-up position), a simple model that captures the geometry of a human's straight body comprised of two neighbouring ellipses touching each other and sharing the same vertical axis, as shown in Fig. 1 can be

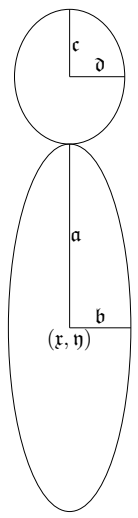

Figure 1. The model used for single human targets in the prescribed application of this paper.

used. With this modelling formalism, the target state space $\mathbb{X}$ is eight-dimensional, and each unlabelled single-target state is

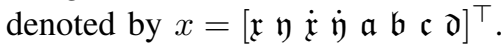

We note that as long as the likelihood function is formulated in a separable form of (10), there is no need to specify the term $f(y)$, because it does not appear in the update equations (15) and (16), and only the single-target-dependent term $g_{y}(\cdot)$ needs to be formulated.

\section{B. Measurement likelihood - shape}

We use a Canny edge detector on each frame $y$. Given a set of targets states (hence their ellipses are given), for each hypothesised target, the shortest distance from every edge pixel to the particular hypothesised double-ellipse structure of the target appearance model is computed. For a valid hypothesis, we expect some of the edge pixels (inlier pixels) to be very close to the prescribed double-ellipse structure. We use the Modified Selective Statistical Estimator (MSSE) [1] algorithm to separate the inlier pixels from outliers, and estimate the mean of squared inlier distances. For the hypothesised target with state $x_{i}$, this mean distance is denoted by $\sigma_{y}^{2}\left(x_{i}\right)$.

One would expect, the likelihood of image measurement $y$ for the given multi-target state $\boldsymbol{X}=\left\{\left(x_{i}, \ell_{i}\right)\right\}_{i=1}^{n}$ to be large when all the mean distances are small. Mathematically, we can express this by:

$$
g_{f e}(y \mid \boldsymbol{X}) \propto \prod_{i=1}^{n} \exp \left[-\alpha \sigma_{y}^{2}\left(x_{i}\right)\right],
$$

where $\alpha$ is a user-defined constant. We note that the proportionality factor is independent of the target states. Therefore, the above likelihood function conforms with the separable form of interest presented in equation (10).

\section{Measurement likelihood - colour}

One of the well established methods to compute colour likelihoods is histogram matching. Following [2], [8], [11], [26], we use kernel density estimation over a set of 500 training HSV histograms denoted by $\left\{h_{j}\right\}_{j=1}^{500}$. As shown in [11], this approach leads to a separable likelihood of the 
form (10) in which the single-target dependent term, $g_{y}$, for a target with HSV histogram $h_{i}$ can be calculated as follows:

$$
g_{f c}(y \mid \boldsymbol{X}) \propto \prod_{i=1}^{n} \frac{\epsilon}{n \times b^{N}} \sum_{j=1}^{n} \stackrel{\circ}{k}\left(\frac{d\left(h_{i}, h_{j}\right)}{b}\right),
$$

where $\stackrel{\circ}{k}$ is the kernel function, $b$ is the kernel bandwidth, $N$ is the number of bins in each histogram and $d\left(h_{i}, h_{j}\right)$ is the Bhattacharyya distance between the histograms [23], [24], [26]. We used Gaussian kernels in our experiments. Since most of the colour features are present in the safety vests, we only use the HSV colour histograms of the pixels of the upper half of the lower ellipse of each target state.

\section{SMC IMPLEMENTATION}

Due to space constraints, we refer the interested reader to [28] for further details on the SMC implementation.

According to Vo et al. [34], if the measurements contents are independent (such as in this work), a sequential update (first by shape and then by colour likelihood) is theoretically equivalent to a single update step with combined likelihood function. Thus, we compute the colour likelihood for each target $i$ using the equation (18) and the weights of particles of each hypothesised target are updated accordingly. Then, in a particle pruning step, we discard the particles with weights less than a small threshold that is adaptively determined as 1.5 times the smallest particle weight.

The retained particles now have a high probability to represent a target and they are then used in the second update step using edge likelihood given in (17). Thus, the algorithm avoids the calculation of the edge likelihoods (which is computationally demanding) of particles which are unlikely to represent a target. This is because with edge likelihood, for each target hypothesis (particle), the distances from all the numerous edge pixels to the hypothesised double-ellipse outline of the target need to be computed then sorted then processed with the MSSE algorithm. Pruning the particles at the first update step leads to substantial savings in the computational burden involved in the second update step, yet maintaining high tracking accuracy.

\section{A. Techniques to Guarantee Computational Tractability}

The particles of each Bernoulli component in the LMB posterior are resampled and components with small probabilities of existence less than a user defined threshold $r_{\text {th }}$ are discarded. Finally, Bernoulli components which overlap more than a user defined threshold (set to 60\%) are merged. The overlapping ratio is the ratio of the area of intersection between two doubleellipse shapes, to the area of the smaller double-ellipse shape. The existence probability of the resultant Bernoulli component is set to be the minimum between 0.999 and the addition of the existence probabilities of the two merged components. The resultant Bernoulli component has all the particles of two merged Bernoulli components. The particle weights are scaled according to the probabilities of existence, and they sum up to 1. The label of the two merged labeled Bernoulli components is chosen to be the label of the older component.

\section{B. State Extraction}

Following the common technique [9]-[11], [28], [34] to estimate the number and states of targets, the labeled Bernoulli components whose probabilities of existence larger than a user-defined application specific threshold $\varepsilon$ are first extracted. For each selected component, the state estimate is then given by the mean of the associated density which can be directly computed as weighted average of its particles:

$$
\hat{\boldsymbol{X}}=\left\{\left(\bar{x}_{\ell}, \ell\right): r^{(\ell)}>\varepsilon\right\}
$$

where $\varepsilon$ is the user defined threshold and $\bar{x}_{\ell}=\sum_{j} w_{\text {up. }}^{(\ell, j)} x_{+}^{(\ell, j)}$. Here $w_{\text {up. }}^{(\ell, j)}$ is the updated weight of the $j$-th particle of the $\ell$-th labeled Bernoulli component. It should be noted that resampling, merging and pruning steps precede the inference step.

\section{Simulation Results}

We implemented our tracking algorithm in MATLAB installed in a standard laptop. The target states models described in section (III-A) are implemented in such a way that they have variable major and minor axis lengths, constrained between a minimum and a maximum. The rationale behind this is that, this will enable the representation of target size variations due to their movements towards and away from the camera.

We set the survival probability of the targets to a constant $p_{S, k}(\cdot)=0.99$ throughout our simulations. As a result of the fact that workers working in an industrial environment are only allowed to walk through designated paths, their direction of motion and the speed are almost constant. Thus we use a nearly constant velocity model to model the movements of the workers.

The birth process is simply chosen to have a single Bernoulli component, hypothesising that with a constant probability of 0.02 , one target may appear at each time step with its location uniformly distributed within the image. In our simulations we use a maximum of $L_{\max }=500$ and a minimum of $L_{\min }=$ 100 particles per target to strike a balance between the tracking accuracy and the computational load.

To evaluate the accuracy of our tracker, we use the well known Multiple Object Tracking Accuracy and Precision (MOTA and MOTP) metrics [14]. The MOTP metric is designed to quantify the consistency of tracked targets with the ground truth and is defined as:

$$
\text { MOTP }=\frac{\sum_{i, k} d_{k}^{i}}{\sum_{k} c_{k}}
$$

where $c_{k}$ is the number of matches found for time step $k$ and $d_{i}$ is the distance between the target $x_{i}$ and its corresponding hypothesis $h_{i}$. The MOTA metric is a combined measure of the number of false positives, detections, and identity switches throughout the tracking period. It is defined by:

$$
\text { MOTA }=1-\frac{\sum_{k}\left(m_{k}+f p_{k}+m m e_{k}\right)}{\sum_{k} g_{k}}
$$


Table I

TRACKING PERFORMANCE FOR OUR DATASET

\begin{tabular}{ccc}
\hline Sequence & MOTA $\%$ & MOTP $\%$ \\
\hline 01 & 72.63 & 86.28 \\
02 & 71.41 & 80.20 \\
03 & 65.79 & 83.13 \\
\hline
\end{tabular}

where $m_{k}, f p_{k}, m m e_{k}$ and $g_{k}$ are the number of misses, false positives, mismatches and number of ground truth objects at time $k$, respectively. These metrics have widely used in the visual tracking literature [14], [33].

We created a dataset of three video sequences recorded in an indoor industrial environment at the RMIT University Robotics lab. This environment was specifically chosen as it closely represent an industrial facility; it is rich in various visual features such as edges and corners, and particularly includes large sections of yellow colour that is similar to the safety vest colour (see Fig. 2). In each video sequence, there are four human targets, three of which are wearing the high visibility vest. One moves randomly, specifically across the camera field of view and the other two (wearing high visibility vests) move as a group and then split, representing the different types of motions that can be present in a real world scenario. The person without the high visibility vest also moves randomly.

The tracking results are given in Table I. The high MOTA and MOTP values presented, attest to the excellent performance of our proposed tracker.

It can be seen in Fig. 2, that our tracker performs accurately in cases where the targets are moving close to each other. This demonstrates the accuracy of the merging step of our tracker. Furthermore all the three sequences depicted in Fig. 2 include a target having a different motion to the other targets, that has been successfully captured by our tracking algorithm. The tracking videos are publicly available ${ }^{2}$.

\section{CONCLUSION}

In this work we formulated two application specific separable likelihood functions that capture the geometric shape and colour information of human workers who are wearing a high visibility (luminous yellow colour) vest. These likelihoods are then used in a labeled multi-Bernoulli filter (implemented using SMC techniques) with a novel two step Bayesian update which guarantees lower computational cost (compared to that of a combined likelihood), yet high tracking accuracy. Preliminary tracking results on a dataset created by the authors are presented with MOTA and MOTP visual tracking benchmark metric values, which are promising.

\section{ACKNOWLEDGEMENT}

This work was supported by ARC Discovery Projects grant DP130104404, and ARC Linkage Projects grant LP130100521.

\footnotetext{
${ }^{2}$ https://www.youtube.com/playlist?playnext=1\&list= PL0Tbf4DdMXCOQ-_sZJoPF7uP7nqY_9TXu
}

\section{REFERENCES}

[1] A. Bab-Hadiashar and D. Suter, "Robust segmentation of visual data using ranked unbiased scale estimate," Robotica, vol. 17, no. 06, pp. 649-660, 1999.

[2] A. Banerjee and P. Burlina, "Efficient particle filtering via sparse kernel density estimation," Image Processing, IEEE Transactions on, vol. 19, no. 9, pp. 2480-2490, 2010.

[3] S. Benhimane and E. Malis, "Real-time image-based tracking of planes using efficient second-order minimization," in Intelligent Robots and Systems, 2004.(IROS 2004). Proceedings. 2004 IEEE/RSJ International Conference on, vol. 1. IEEE, 2004, pp. 943-948.

[4] M. J. Black and A. D. Jepson, "Eigentracking: Robust matching and tracking of articulated objects using a view-based representation," International Journal of Computer Vision, vol. 26, no. 1, pp. 63-84, 1998.

[5] D. Comaniciu, V. Ramesh, and P. Meer, "Kernel-based object tracking," Pattern Analysis and Machine Intelligence, IEEE Transactions on, vol. 25 , no. 5, pp. 564-577, 2003.

[6] L. J. Cox and S. L. Hingorani, "An efficient implementation of reid's multiple hypothesis tracking algorithm and its evaluation for the purpose of visual tracking," Pattern Analysis and Machine Intelligence, IEEE Transactions on, vol. 18, no. 2, pp. 138-150, 1996.

[7] S. Dambreville, Y. Rathi, and A. Tannenbaum, "Tracking deformable objects with unscented kalman filtering and geometric active contours," in American Control Conference, 2006. IEEE, 2006, pp. 6-pp.

[8] A. Elgammal, R. Duraiswami, and L. S. Davis, "Efficient kernel density estimation using the fast gauss transform with applications to color modeling and tracking," Pattern Analysis and Machine Intelligence, IEEE Transactions on, vol. 25, no. 11, pp. 1499-1504, 2003.

[9] R. Hoseinnezhad, B.-N. Vo, D. Suter, and B.-T. Vo, "Multi-object filtering from image sequence without detection," in Acoustics Speech and Signal Processing (ICASSP), 2010 IEEE International Conference on. IEEE, 2010, pp. 1154-1157.

[10] R. Hoseinnezhad, B.-N. Vo, and B.-T. Vo, "Visual tracking in background subtracted image sequences via multi-bernoulli filtering," Signal Processing, IEEE Transactions on, vol. 61, no. 2, pp. 392-397, 2013.

[11] R. Hoseinnezhad, B.-N. Vo, B.-T. Vo, and D. Suter, "Visual tracking of numerous targets via multi-bernoulli filtering of image data," Pattern Recognition, vol. 45, no. 10, pp. 3625-3635, 2012.

[12] M. Jenkin, N. Bains, J. Bruce, T. Campbell, B. Down, P. Jasiobedzki, A. Jepson, B. Majarais, E. Milios, S. Nickerson et al., "Ark: autonomous mobile robot for an industrial environment," in Intelligent Robots and Systems' 94.'Advanced Robotic Systems and the Real World', IROS'94. Proceedings of the IEEE/RSJ/GI International Conference on, vol. 2. IEEE, 1994, pp. 1301-1308.

[13] S. J. Julier and J. K. Uhlmann, "New extension of the kalman filter to nonlinear systems," in AeroSense' 97. International Society for Optics and Photonics, 1997, pp. 182-193.

[14] B. Keni and S. Rainer, "Evaluating multiple object tracking performance: the clear mot metrics," EURASIP Journal on Image and Video Processing, vol. 2008, 2008.

[15] T. J. Larsson et al., "Industrial forklift trucks: Dynamic stability and the design of safe logistics," Safety Science Monitor, vol. 7, no. 1, 2003.

[16] D. Lecking, O. Wulf, V. Viereck, J. Tödter, B. Wagner et al., "The rts-still robotic fork-lift," EURON Technology Transfer Award, 2005.

[17] P. Li, T. Zhang, and B. Ma, "Unscented kalman filter for visual curve tracking," Image and vision computing, vol. 22, no. 2, pp. 157-164, 2004.

[18] R. P. Mahler, "Multitarget bayes filtering via first-order multitarget moments," Aerospace and Electronic Systems, IEEE Transactions on, vol. 39, no. 4, pp. 1152-1178, 2003.

[19] — - Statistical multisource-multitarget information fusion. Artech House, Inc., 2007.

[20] - Advances in Statistical multisource-multitarget information fusion. Artech House, Inc., 2014.

[21] I. Matthews, T. Ishikawa, and S. Baker, "The template update problem," IEEE Transactions on Pattern Analysis \& Machine Intelligence, no. 6, pp. 810-815, 2004.

[22] H. Nanda and L. Davis, "Probabilistic template based pedestrian detection in infrared videos," in IEEE Intelligent Vehicle Symposium, vol. 1, 2002, pp. 15-20.

[23] K. Nummiaro, E. Koller-Meier, and L. Van Gool, "Object tracking with an adaptive color-based particle filter," in Pattern Recognition. Springer, 2002, pp. 353-360. 



Figure 2. Screen shots of tracking results. Each row is from one sequence.

[24] K. Okuma, A. Taleghani, N. De Freitas, J. J. Little, and D. G. Lowe, "A boosted particle filter: Multitarget detection and tracking," in Computer Vision-ECCV 2004. Springer, 2004, pp. 28-39.

[25] J. A. Palazon, J. Gozalvez, J. L. Maestre, and J. R. Gisbert, "Wireless solutions for improving health and safety working conditions in industrial environments," in e-Health Networking, Applications \& Services (Healthcom), 2013 IEEE 15th International Conference on. IEEE, 2013, pp. 544-548.

[26] P. Pérez, C. Hue, J. Vermaak, and M. Gangnet, "Color-based probabilistic tracking," in Computer vision-ECCV 2002. Springer, 2002, pp. 661-675.

[27] C. Rasmussen and G. D. Hager, "Joint probabilistic techniques for tracking multi-part objects," in Computer Vision and Pattern Recognition, 1998. Proceedings. 1998 IEEE Computer Society Conference on. IEEE, 1998, pp. 16-21.

[28] T. Rathnayake, A. K. Gostar, R. Hoseinnezhad, and A. Bab-Hadiashar, "Labeled multi-bernoulli track-before-detect for multi-target tracking in video," in Information Fusion (Fusion), 2015 18th International Conference on. IEEE, 2015, pp. 1353-1358.

[29] S. Reuter, B.-T. Vo, B.-N. Vo, and K. Dietmayer, "The labeled multibernoulli filter," Signal Processing, IEEE Transactions on, vol. 62, no. 12, pp. 3246-3260, 2014.

[30] J. L. Ryan and L. D. Ryan, Forklift and Stacker Manual, United States: Donegal Bay Incorporated Publishing, 2006.

[31] S. Saric, A. Bab-Hadiashar, R. Hoseinnezhad, and I. Hocking, "Analysis of forklift accident trends within victorian industry (australia)," Safety Science, no. 60, pp. 176-184, 2013.

[32] M. Sepulcre, J. A. Palazon, J. Gozalvez, and J. Orozco, "Wireless connectivity for mobile sensing applications in industrial environments," in Industrial Embedded Systems (SIES), 2011 6th IEEE International Symposium on. IEEE, 2011, pp. 111-114.

[33] R. Stiefelhagen, K. Bernardin, R. Bowers, J. Garofolo, D. Mostefa, and P. Soundararajan, "The clear 2006 evaluation," in Multimodal Technologies for Perception of Humans. Springer, 2007, pp. 1-44.

[34] B.-N. Vo, B.-T. Vo, N.-T. Pham, and D. Suter, "Joint detection and estimation of multiple objects from image observations," Signal Processing, IEEE Transactions on, vol. 58, no. 10, pp. 5129-5141, 2010.

[35] B.-N. Vo, B.-T. Vo, and D. Phung, "Labeled random finite sets and the bayes multi-target tracking filter," 2013.

[36] B.-T. Vo and B.-N. Vo, "Labeled random finite sets and multi-object conjugate priors," Signal Processing, IEEE Transactions on, vol. 61, no. 13 , pp. $3460-3475,2013$.

[37] B.-T. Vo, B.-N. Vo, and A. Cantoni, "Analytic implementations of the cardinalized probability hypothesis density filter," Signal Processing, IEEE Transactions on, vol. 55, no. 7, pp. 3553-3567, 2007.

[38] _ , "The cardinality balanced multi-target multi-bernoulli filter and its implementations," Signal Processing, IEEE Transactions on, vol. 57, no. 2, pp. 409-423, 2009.

[39] L. Zhu, J. Zhou, and J. Song, "Tracking multiple objects through occlusion with online sampling and position estimation," Pattern Recognition, vol. 41 , no. 8 , pp. $2447-2460,2008$ 